\title{
Ergonomics Risk Factors among Hairdressers Working in India: A Case Study
}

\author{
Mishra $\mathbf{A}^{1}$, Singh $\mathbf{R}^{1}$ and Tandon $\mathbf{P}^{2 *}$ \\ ${ }^{1}$ Delhi Technological University, India \\ ${ }^{2}$ IIITDM, Jabalpur, India
}

*Corresponding author: Puneet Tandon, IIITDM, Jabalpur, India, Tel: +91-94253-24240;

Email: ptandon@iiitdmj.ac.in
Case Report

Volume 4 Issue 4

Received Date: July 20, 2020

Published Date: August 10, 2020

DOI: $10.23880 /$ eoij-16000252

\section{Abstract}

Hairdressers in India are at a high risk of work-related musculoskeletal disorders (MSD) for various reasons, including holding scissors, awkward postures, repetitive tasks, etc. This case study is to investigate the intensity of MSD among the hairdressers in India. Musculoskeletal disorders have become a rather frequent subject of dissatisfaction among cosmetologists. The objective of this study was to identify the grey areas, where the risk is involved during the entire work cycle for a hairdresser. Also, it brings light to the workplace-related issues faced by hair salon employees and customers due to the current practices relevant to Indian hair salons, mainly considering the global pandemic. This study also discusses the broad spectrum of these salons, how they lie on the economic scale, and the evaluation of their working environments. The postural assessments have been carried out with the help of Rapid Entire Body Assessment (REBA) and Rapid Upper Limb Assessment (RULA), which are the tools to gauge and evaluate the ergonomic risk factors associated with an individual's task. The observations and inferences drawn from this assessment were used to analyze the workplace-related hazards for the professionals involved in the entire process of a haircut. These results were also used to make suggestions and design interventions to make the working environment more ergonomic and suitable.

Keywords: Physical Ergonomics; Musculoskeletal Injury; REBA; RULA

Abbreviations: WRMSDs: Work-Related Musculoskeletal Disorders; REBA: Rapid Entire Body Assessment; RULA: Rapid Upper Limb Assessment.

\section{Background}

Work-related musculoskeletal disorders (WRMSDs) are one of the severe occupational health issues within the workers worldwide [1]. Previous studies have identified inappropriate workspace and associated tools, which force the workers to adopt awkward postures for more extended periods, which leads to the occurrence of WRMSDs [2]. These risk factors reduce the overall productivity of the workers and decrease the quality of work as well. Mostly in rural India, some barbers do haircutting under the tree; they do not have a proper workstation for customers and themselves.
For the majority of the people in rural and urban India, roadside hairdressing under the tree is one of the means of livelihood, and it is solely dependent on human labour [3]. This also showcases that there is a huge potential for design intervention. Hairdresser jobs require both physical as well as mental efforts to satisfy the customer(s). Hairdressers or hairstylists are exposed to various hazards in the workplace, such as awkward posture, i.e., bending neck, twisting wrist, load on muscles, and prolonged standing. They are required to use a variety of tools that need engagement from their hands, sometimes even in odd positions. Different segments of workers are discovered at risk of progressing WRMSDs [4], including; postman, assembly line workers, call center employees, and other administrative workers [5]. A literature review revealed a few studies describing the incremental growth of WRMSDs in the hair cutting 
sector and the importance of monitoring and addressing injuries through design intervention in the workstations of hairdressers. However, all of these studies were carried out from the perspective of western countries, like the US and EU, where the working conditions differ greatly with that of India [6]. Besides, haircutting is considered to be taboo as only marginalized community people are involved in this profession. As a result of this, no standards for workstations exist for the marginalized hairdressers. In most of India, mechanization has not yet been accepted in this profession, and mostly hairdressers run their business from unhygienic small shops or under the tree. Most of the reasonably inexpensive Indian salons or parlours employ young teenagers for various jobs because of a variety of reasons. However, the general age of hairdressers' range from anywhere around 18 to 40, both males and females. The effects of negligence to their postures can accumulate over the years and pose long term health risks by the time these professionals age and even result in musculoskeletal disorders. This problem has become graver due to the impact of novel COVID-19 and the need to maintain social distancing, which is almost impossible, with the current designs of nonexistent workstations.

\section{Case Study}

The case study was conducted to investigate the magnitude of MSD among the hairdressers. Ergonomics analysis of the work of hairdressers was carried out with the help of Rapid Entire Body Analysis (REBA) and Rapid Upper Limb Analysis (RULA) techniques [3]. Both the techniques are majorly used to evaluate the risk of MSDs for the assessment of working postures in hair salons [7]. The study involved two tasks for evaluation, hair wash, and haircut. For most of the activities carried out at salons, the hairdresser must start right from the hair wash. A pattern was observed during the direct observation when a customer walks in or check-in for a haircut. There are two possibilities. They either had their hair washed followed by a haircut or simply just got a haircut. The whole process usually consists of various subtasks, i.e., washing the hair and preparing it for a cut, the hair cutting process, and the hair drying and styling process.

\section{Hair Wash}

The task of washing and preparing the customers' hair for a cut is handled by one individual and is the first step in the entire process of a haircut (Figure 1). It was essential to pay attention to the deviations from the neutral posture of the professionals involved in this task. The individual uses his right hand to hold the showerhead and left hand to lather or rinse the product in the customers' hair and vice-versa as per the preferences. The assessment of their body posture has been carried out through the REBA and RULA analysis, and while drawing the conclusions, basic ergonomic principles have been kept in mind. The entire assessment has been divided into three sections, to ensure proper attention is given to all the major body parts and positions.

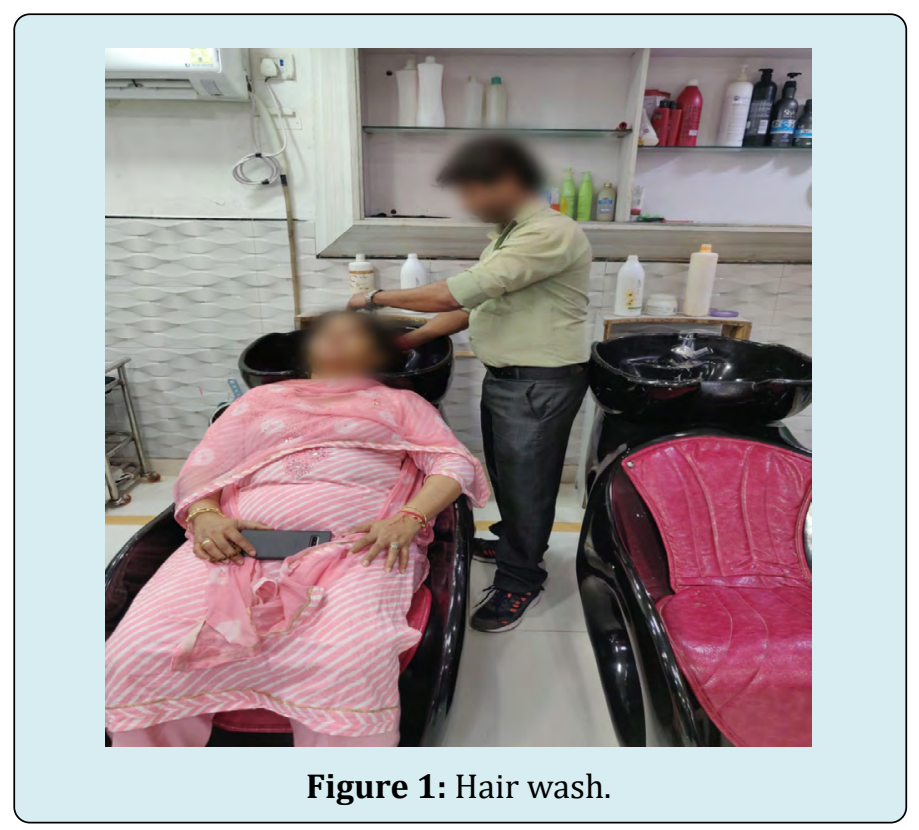

\section{Arm and Wrist Analysis}

The upper arm was being used at an angle roughly between $45^{\circ}-90^{\circ}$, along with raised and unrelaxed shoulders, as shown in Figure 2a. The lower arms were also being used in extreme ranges, in addition to the static load being offered by the showerhead. The wrist movement involved a lot of twisting and bending, because of the nature of the task and equipment. This also makes the arms work across the midline of the body. The non-neutral position of wrists and arms and the repetitive motion add to the excessive load and discomfort.

\section{Neck and Trunk Analysis}

It was observed that during the task, the neck and spine were not aligned and excessive stress and the load was affecting the neck area (Figure 2b). The neck was being extended at $+20^{\circ}$, along with being bent slightly to the side. This was mainly because of a lack of clearance and reachability. Poor posture also contributed to a slouching back and further stress in the lower back. 


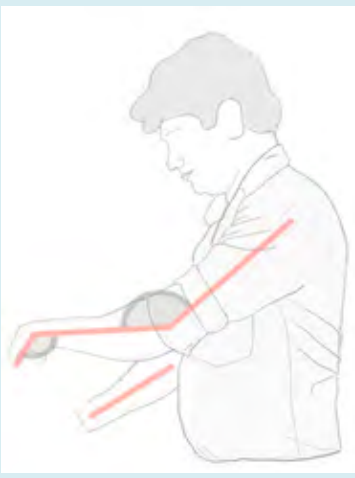

a

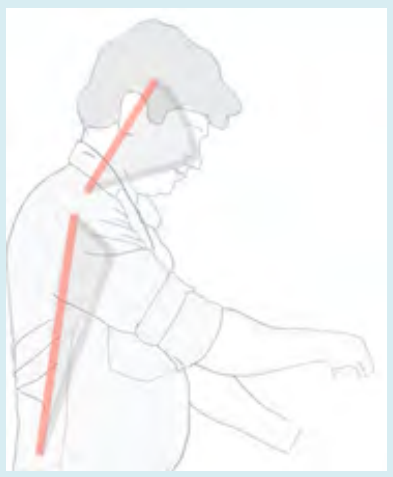

b

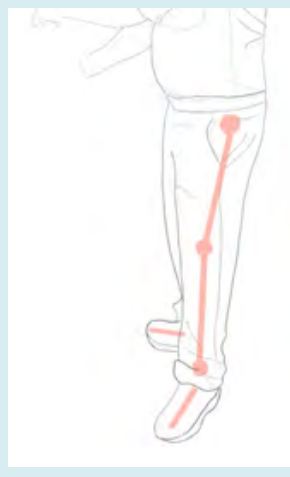

C

Figure 2: Posture analysis of hair wash.

\section{Lower Body Analysis}

The body weight of the individual was not distributed equally among the legs because of the lack of any possible support, as shown in Figure 2c. Standing for over 7-8 minutes at a time becomes a source of fatigue and discomfort. The low clearance in surroundings also forces him to stand in awkward positions.

In conclusion, due to lack of clearance, the employee had problems in equal bodyweight distribution, keeping his torso aligned with his back, and maintaining a neutral position of arms and wrists. A lot of the joints were not in neutral positions and were ignored completely. The restricted body movement leads to the usage of excessive force and static load. After analyzing the postures associated with the hair wash, it was observed that the corresponding scores of REBA and RULA were 11 and 7 (Appendix I and II), respectively, which is on the end of the spectrum and the risk involved is very high in this scenario.

\section{Hair Cut}

The second and most prominent step in the process is the actual task of cutting the hair. Major deviations from the essential neutral posture were observed. The hairdresser spent about 10-20 minutes on the participants' moderately long hair. The tools used by the hairdresser consisted of the usual metal scissors, combs, hairdryers, and other styling equipment. During this assessment, multiple observations were made about the posture and later validated through the REBA and RULA analysis.

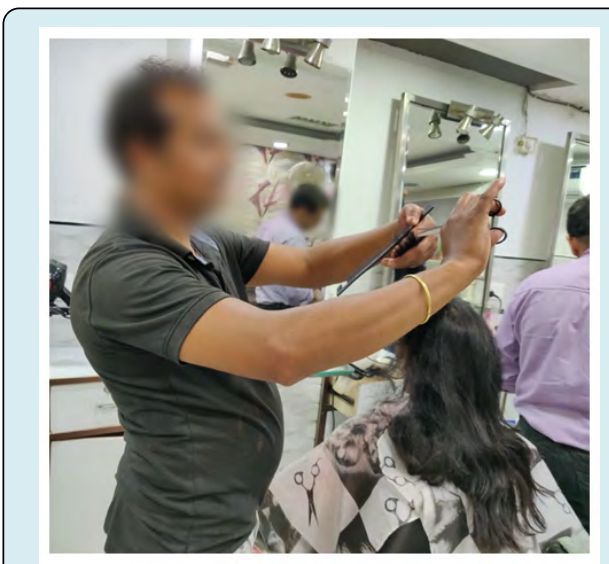

Figure 3: Hair-cut.

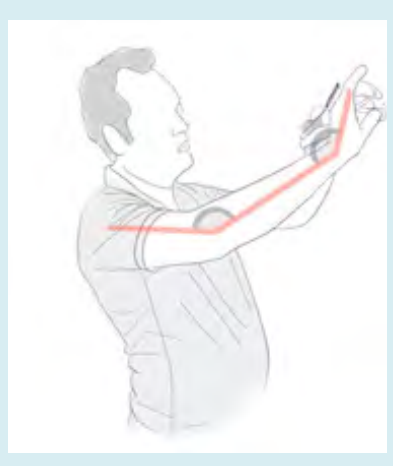

a

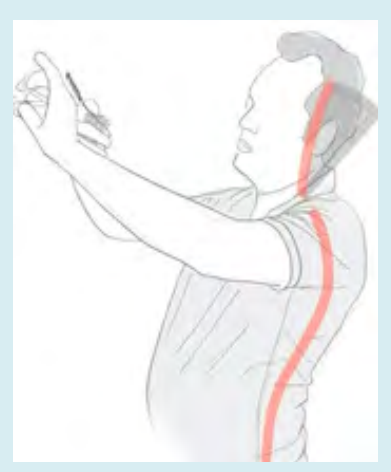

b

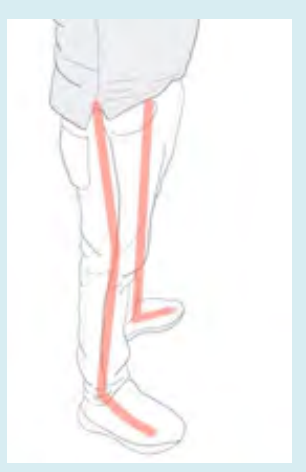

C

Figure 4: Posture analysis of hair cutting. 


\section{Arm and Wrist Analysis}

The arms are at an extreme angle of above $90^{\circ}$ with raised shoulders and flexed wrists, as shown in Figure $4 \mathrm{a}$. The position of the upper and lower arms creates a lot of static loads and leads to fatigue. The repetitive nature of the task also adds to discomfort and weariness. While the grip of the scissors and comb reduces the strain on the neck, it is unacceptable since the shoulders, elbow, and wrists come far from their neutral position.

\section{Neck and Trunk Analysis}

The neck is extended backward between $10^{\circ}$ to $20^{\circ}$ along with being slightly bent to one side. The trunk is also extended to the back which puts a lot of pressure on the lower back. Standing like this for 10-15 minutes while working on a haircut, may lead to a lot of back-related MSD's. As visible in the illustration (Figure 4b), the back of the individual is already starting to seem inverted.

\section{Lower Body Analysis}

The bodyweight of the individual was not distributed equally among the legs because of the lack of any support, as shown in Figure 4c. Standing for over 7-8 minutes at a time becomes a source of fatigue and discomfort. The low clearance in surroundings also forces him to stand in awkward positions.

In conclusion, the static load on the arms and wrists is unacceptable and unnecessary. The arm joints are not in a neutral position and suffer undue amounts of stress. While the worker attempts to not strain his neck, in the bargain, he has to arch his back and exert pressure on his lower back. The wrists are also made to bend at undesirable angles, and these postural habits result in a musculoskeletal disorder. The corresponding REBA and RULA scores for the postures came out to be 12 and 7 (Appendix III and IV) respectively. These scores are at the extreme ends of the scale and suggest that immediate change needs to be implemented in these high risk posing scenarios [8].

\section{Conclusion}

The objective of this study was to evaluate the workplacerelated ergonomic risks faced by the hairdressers involved in the task of hair washing and cutting, and this was assessed with the help of REBA and RULA assessment. The inferences drawn from the analysis suggested that clearance in the workspace played a significant role in the poor working posture adopted by the hairdressers. Due to a lack of proper environment and equipment, they had to compromise on their posture, which may not seem like a problem at the initial stage, but later it becomes a significant issue for the hairdressers. It was also observed that the workers accustomed to their surroundings, moulded themselves according to the equipment available and indulged in poor postural habits in the process. The REBA and RULA analysis helped in assessing the ergonomic risk involved in the aforementioned processes, and the scores derived in this scenario were at the extreme ends of the spectrum. The REBA score for the hair washing process was 11, and the RULA score came out to be a 7 . This suggests very high risk, and immediate change is recommended to be implemented. The REBA score for the haircutting process was 12, and the RULA process was a 7. Like the hair washing process, this task also poses a very high risk and requires immediate change. The remarkable similarity in the score and risk levels suggests that the working environment was completely ignored by the hairdressers' point of view. The first and foremost change that can be brought out in the workspace environment would be to increase clearance so workers can move about freely and not be restricted in motion. This would solve issues where workers are forced to be in undesirable postures due to a lack of space for comfortable movement. The shampoo stations in the current set were built too close together. Since the jobs of hairdressers are very physically demanding, the working environment must be built according to their needs. Standing chairs can also be employed for more static jobs, as the discomfort levels while standing is higher than when sitting. The support offered by the chairs will also ensure proper weight distribution, and a better back and neck posture. The tasks of long and static jobs of shampooing and hair cutting can drastically be made less physically challenging with this intervention. Ergonomically designed tools and equipment will also help reduce ergonomic risks. Salons can use ergonomic scissors, combs, dryers, showerheads, etc. to ensure the long hours that the workers spend using them, does not end up hurting or posing any future risk to their health. This need for the ergonomic workstation, equipment, and space for beauty parlours and salons has become all the more important due to the new normal that we have to practice in light of the global pandemic.

\section{References}

1. Kattel BP (1996) The effect of upper-extremity posture on maximum grip strength. International Journal of Industrial Ergonomics 18(5-6): 423-429.

2. Kumar S (2001) Theories of musculoskeletal injury causation. Ergonomics 44(1): 17-47.

3. Hignett S (2000) Rapid entire body assessment (REBA). Applied Ergonomics, pp: 201-205.

4. Luopajarvi T (1979) Prevalence of tenosynovitis and other injuries of the upper extremities in repetitive work. Scandinavian journal of work, environment \\& 
health 3: 48-55.

5. Hocking B (1987) Epidemiological aspects of "repetition strain injury" in Telecom Australia. Medical Journal of Australia 147(5): 218-222.

6. Mukhopadhyay P (2015) The evaluation of ergonomic risk factors among meat cutters working in Jabalpur, India. International Journal of Occupational and Environmental Health 21(3): 192-198.
7. Winkel J (1996) A model for solving work related musculoskeletal problems in a profitable way. Applied Ergonomics 27(2): 71-77.

8. MahdaviS(2013) Evaluation of therisk of musculoskeletal disorders using Rapid Entire Body Assessment among hairdressers in Khorramabad, Iran, in 2014. Journal of Occupational Health and Epidemiology\} 2(3): 138-145. 